\title{
Silver-Russell Syndrome: Orthodontic Perspective
}

\author{
Zahra Khalid, Kashif Iqbal, Abdullah Jan and Asif Khurshid \\ Department of Orthodontics, Armed Forces Institute of Dentistry, Rawalpindi, Pakistan
}

\begin{abstract}
Silver-Russell syndrome (SRS) is a rare disorder characterised by prenatal and postnatal growth deficiency, a relatively large head size with triangular face, a prominent forehead, body asymmetry, feeding difficulties, clinodactyly and other features. Abnormalities of chromosome number 7 and 11 have been found in $60 \%$ of patients, but the diagnosis of the disease is mainly by ascertaining clinical features. We, herein, report a case of an 11-year boy who presented to Orthodontics Department of Armed Forces Institute of Dentistry (AFID), for treatment of craniofacial and oral problems, which included small-sized jaws and severely crowded teeth. The patient is undergoing serial extraction treatment at the moment, which will be followed by further orthodontic treatment.
\end{abstract}

Key Words: Silver-Russell syndrome, Growth deficiency, Triangular face, Body asymmetry.

How to cite this article: Khalid Z, Iqbal K, Jan A, Khurshid A. Silver-Russell Syndrome: Orthodontic Perspective. J Coll Physicians Surg Pak 2020; 30(12):1352-1354 .

\section{INTRODUCTION}

Silver-Russell syndrome (SRS) is a rare disorder occurring in about $1 / 100,000$ births. ${ }^{1}$ The syndrome was first described by Silver and Russell, who independently described a subset of children with pre-natal growth deficiency, post-natal dwarfism and other features, which included low birth weight, feeding problems, hypoglycemia, excessive sweating, weak muscle tone, large head due to a small body size, wide and late-closing fontanelle, clinodactyly, and body asymmetry, as one side of the body grows more slowly than the other. The majority of patients have normal intelligence but motor and speech delay is common. ${ }^{2}$

We, herein, present a case of a boy who visited us with his mother with complaint of severely crowded and crooked teeth. The patient's disease was diagnosed by the pediatrician and was already undergoing growth hormone $(\mathrm{GH})$ therapy. We, present here the general, craniofacial and dental features of the patient to help increase the awareness of the syndrome.

\section{CASE REPORT}

An 11-year male was referred to us by the endocrinologist for the management of crowded teeth. The patient was diagnosed as a case of SRS at the age of two years by the pediatrician. He was born at 38 weeks of gestation with a birth weight of $1.5 \mathrm{~kg}$ and with a twin brother, who was completely normal. There was no positive family history of any genetic or growth disorder.

Correspondence to: Dr. Zahra Khalid, Department of Orthodontics, Armed Forces Institute of Dentistry,

Rawalpindi, Pakistan

E-mail: zahrakhalid26@gmail.com

Received: July 22, 2019; Revised: November 04, 2019;

Accepted: November 17, 2019

DOI: https://doi.org/10.29271/jcpsp.2020.12.1352
The height of the patient at the time of presentation to us was 127 metres. His presenting weight was $15 \mathrm{kgs}$. There was a slight body asymmetry with a short left arm and leg and clindactyly of the fifth digits.

Craniofacial and orthodontic evaluation showed a relatively large-size head, triangular face, low-set and asymmetric ears, mandibular retrognathia and cleft palate. The patient was skeletal class I, high angle case with increased maxilla-mandibular angleand microgenia. Dental findings indicated the patient in the mixed dentition phase with development of severe crowding of more than $10 \mathrm{~mm}$ in both the jaws. Upper left central incisor was buccally ectopic due to lack of space. Similarly, the lower incisors were severely crowded and there was lack of space for the eruption of canines in both the arches. The molar relationship was bilaterally Class I (Figure 1). Periodontal status was satisfactory with normal probing depths and no bleeding on probing.

Dental panoramic examination revealed a dental age of 10 and a disturbed pattern of dental exfoliation with the lower first premolars erupting before the lower canines, thus impeding their eruption. The crowns of second molars were formed and the crypts of third molars could be seen (Figure 2).

The patient was already undergoing GH therapy (Noditropin, 2 units subcutaneous, once daily) for the last 8 years with an increase in height by 8-10 cmevery year.

Orthodontic treatment plan included serial extractions with extraction of deciduous canines, followed by extraction of deciduous first molars and then first premolars. This will be followed by fixed Orthodontic therapy to align and level the arches. The patient is currently at the stage of follow-up for extraction of all first premolars. 


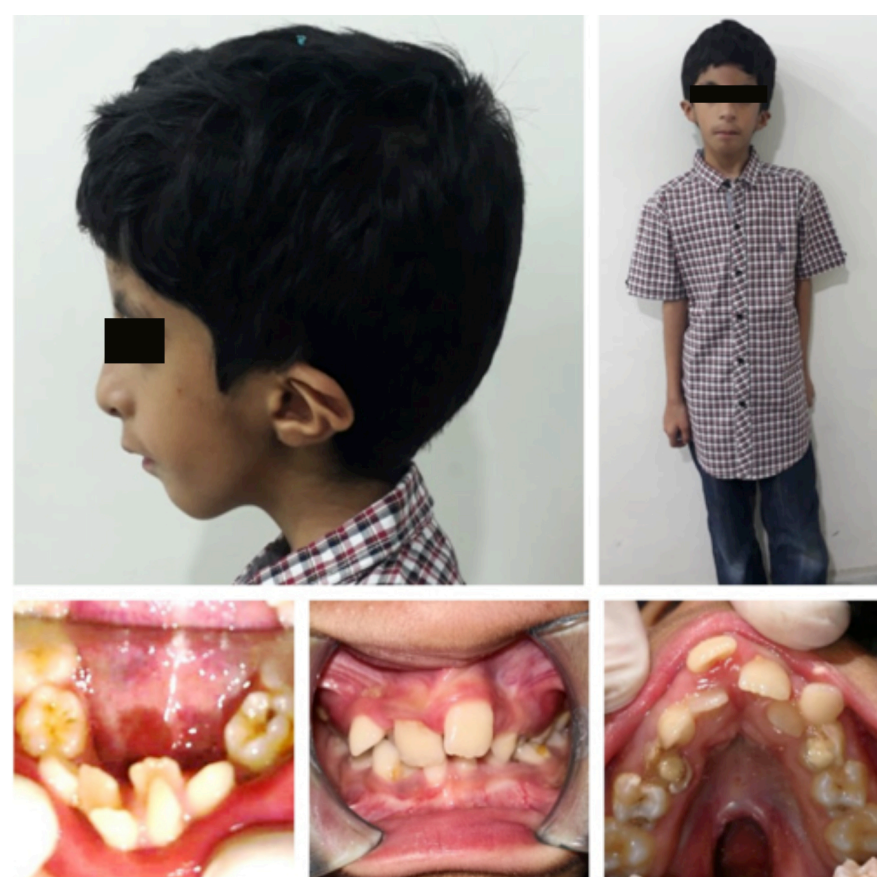

Figure 1: Clinical photographs of the patient and his teeth showing body asymmetry, large size head, triangular face, severe crowding of teeth and cleft palate.

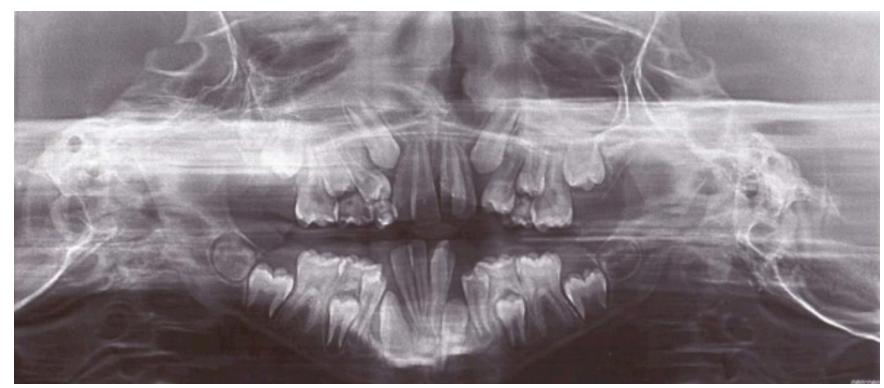

Figure 2: Orthopantomogram of the patient showing abnormal eruption pattern in the mandible.

\section{DISCUSSION}

SRS is a rare but well-recognised condition, associated with prenatal and postnatal growth retardation. The syndrome was first described by Silver and Russell, who independently described a subset of children with low birth weight, postnatal short stature, characteristic facial features and body asymmetry. Almost all patients with SRS are born small for gestational age. The etiology of intrauterine growth retardation is extremely heterogeneous. Children with SRS can be distinguished from those with idiopathic intrauterine growth retardation or small-for-gestational-age (SGA) and postnatal growth failure by the presence of other characteristic features, including relative macrocephaly, prominent forehead, body asymmetry and feeding difficulties, fifth finger clinodactyly, low muscle mass, speech delay, low-set ears, hypoglycemia, scoliosis, micrognathia and crowded teeth. ${ }^{2,3}$

Craniofacial features include frontal bossing, reduced vertical facial proportions, triangular face with a narrow and small mandible with short ramus and down-turned corners of the mouth (sharks mouth), low-set posteriorly rotated ears. The dental manifestations include microdontia, high-arched palate and severe crowding due to small-sized jaws, mandible, in particular. ${ }^{3}$

An underlying molecular cause can be identified in about $60 \%$ of patients, the most common mechanism being the loss of methylation on chromosome 11p15 and maternal uniparental disomy for chromosome 7. The diagnosis is mainly clinical with patients scoring at least 4 out of 6 criteria of the Netchine-Harbison Clinical scoring system being confirmed for diagnosis, even if moleculartests are normal. ${ }^{1}$

Management of SRS requires a multidisciplinary approach with paediatric subspecialists such as an endocrinologist (co-ordinator), gastroenterologist, dietician, clinical geneticist, craniofacial team, orthodontists, orthopaedic surgeon, neurologist, speech and language thera pist and psychologist as members of the team. ${ }^{1,2,4}$

Early feeding and nutritional support is very important to address low birth weight, low muscle mass and poor appetite. Monitoring of levels of urinary ketones is usually effective in preempting hypoglycemia related to fasting, activity or illness.

GH treatment is required to address stunted growth. A satisfactory growth response and an increase in predicted adult height of 7-11 cm at pharmacological doses of GH has been demonstrated. Body asymmetry and scoliosis need to be addressed by the orthopedic surgeon.

Palatal clefts should be addressed early around 12-18 months of age to help in speech development. Crowding can be addressed by procedures like serial extraction, maxillary expansion or symphyseal distraction. Serial extraction involves planned extraction of deciduous teeth followed by some permanent teeth in an orderly sequence to guide the permanent teeth in a more favourable position. Maxillary expansion can also gain space in mild cases of crowding. Mandibular expansion or symphyseal distraction can also be used to gain space in a collapsed mandibulararch.

SRS is manageable, especially if treatment is started early. GH therapy can help the child grow more rapidly, but still the child will be shorter than the average. A multidisciplinary approach results in better treatmentoutcomes:

\section{PATIENT'S CONSENT:}

Informed consent was obtained from the patient and the parent for publishing the case report.

\section{CONFLICT OF INTEREST:}

The authors declared no conflict of interest.

\section{AUTHORS'CONTRIBUTION:}

ZK: Drafted the manuscript, diagnosed and formulated the treatmentplan.

$\mathrm{KI}$ : Drafted the manuscript and treated the patient.

AJ: Supervised the reportand the case.

AK: Analysed the patient's records, and treating the case. 


\section{REFERENCES}

1. Wakeling EL, Brioude F, Lokulo-Sodipe O, O'Connell SM, Salem J, Bliek J, et al. Diagnosis and management of Silver-Russell syndrome: First international consensus statement. Nat Rev Endocrinol 2017; 13(2):105-24. doi: 10.1038/nrendo.2016.138.

2. Azzi S, Salem J, Thibaud N, Chantot-Bastaraud S, Lieber E, Netchine I, et al. A prospective study validating a clinical scoring system and demonstrating phenotypicalgenotypical correlations in Silver-Russell syndrome. J Med
Genet 2015; 52(7):446-53. doi: 10.1136/jmedgenet-2014102979.

3. Ko SJ, Seo JY, Kwon YD, Cheon K, Park JH. Orthodontic treatment in conjunction with twin-bock treatment and growth hormone therapy in silver russell syndrome. J Clin Pediatr Dent 2017; 41(5):392-7. doi: 10.17796/1053-462841.5.392.

4. Hodge N, Evans CA, Simmons KE, Fadavi S, Viana G. Occlusal characteristics of individuals with growth hormone deficiency, idiopathic short stature, and Russell-Silver syndrome. J Dent Child (Chic) 2015; 82(3):135-40. 